\title{
Catheter-Directed Thrombolysis in a Patient with Severe COVID-19 Pneumonia on Extracorporeal Membrane Oxygenation
}

\author{
Guy J. Padley, $\mathrm{BSC}^{1,2}$ Shivani S. Desai ${ }^{1,3}$ Chrissy Weaver, MBChB, MRCP, EDICT, FICM ${ }^{4}$ \\ Laura C. Price, MBChB, BSc, PhD, FRCP5 Deepa J. Arachchillage, MRCP, MD, FRCPath $6,7,8$ \\ Carole A. Ridge, FFRRCSI ${ }^{1}$
}

${ }^{1}$ Department of Radiology, Royal Brompton Hospital, London, United Kingdom

2 University of Leeds Medical School, Leeds, United Kingdom

${ }^{3}$ St George's University of London, London, United Kingdom

${ }^{4}$ Anaesthesia and Critical Care, Royal Brompton Hospital, London, United Kingdom

${ }^{5}$ National Pulmonary Hypertension Service, Royal Brompton Hospital, London, United Kingdom

${ }^{6}$ Department of Haematology, Imperial College London, London, United Kingdom

${ }^{7}$ Department of Haematology, Royal Brompton Hospital, London, United Kingdom

${ }^{8}$ Department of Haematology, Imperial College Healthcare NHS

Trust, London, United Kingdom

Semin Thromb Hemost 2020;46:850-852.

Coronavirus disease 2019 (COVID-19) is a highly prothrombotic condition leading to thrombosis in both venous and arterial circulations, including microvascular thrombosis in pulmonary and renal vasculature despite intensified anticoagulation. COVID-19 initiates cellular infection by binding angiotensin-converting enzyme 2 at the surface of host cells, including endothelial cells. ${ }^{1}$ Attachment promotes disordered cytokine paracrine signaling, including proinflammatory molecules, proapoptotic mediators, and activation of coagulation, leading to thrombosis. Coagulation abnormalities are wellknown features of severe infections, but virtually in all reports these changes (and especially thrombosis) are more common in COVID-19 than in other pneumonias despite standard thromboprophylaxis or even with treatment dose anticoagulation. ${ }^{2}$ The reported incidence of thrombosis in patients with COVID-19 varies considerably, depending on anticoagulant regimen and whether the patient was treated in intensive care, due to additional risk factors such as central lines, and immobility and mechanical ventilation. A multicenter study of 150 COVID-19 patients demonstrated a $43 \%$ prevalence of thrombosis, despite prophylactic or therapeutic anticoagulation. ${ }^{2}$ The pathophysiology of thrombosis in COVID-19 is most likely multifactorial and the high rate of thrombosis appears to be driven by endothelial inflammation and elevated coagula-

published online September 4, 2020
Issue Theme Maintaining Hemostasis and Preventing Thrombosis in COVID-19 -Part I; Guest Editors: Emmanuel J. Favaloro, PhD, FFSc (RCPA), and Giuseppi Lippi, MD.
Address for correspondence Deepa R.J. Arachchillage, MRCP, MD, FRCPath, Department of Haematology, Imperial College Healthcare NHS Trust and Imperial College London, Hammersmith Hospital, 4th Floor, Commonwealth Building, Du Cane Road, London W12 ONN, United Kingdom (e-mail: d.arachchillage@imperial.ac.uk).

tion factors such as fibrinogen and factor VIII. ${ }^{2}$ Extracorporeal membrane oxygenation (ECMO), in which gas exchange occurs by means of an extracorporeal membrane perfused with venous blood, is life saving for many patients with severe respiratory failure who do not respond to other methods of supporting oxygenation and ventilation, including mechanical ventilation during the COVID-19 pandemic. However, despite the significantly improved circuits and techniques used for ECMO in recent decades, thrombosis and bleeding are still major complications in patients receiving ECMO support. Therefore, maintaining the fine balance of hemostasis to prevent blood clots in patients and the ECMO circuit while preventing major bleeding complications, especially intracranial bleeding, is vital in this process.

We report a 40-year-old female with severe COVID-19 pneumonia who had catheter-directed thrombolysis on veno-venous ECMO. She was referred to our unit for venovenous ECMO with no improvement despite mechanical ventilation. Her body mass index was $29.4 \mathrm{~kg} / \mathrm{m}^{2}$. She was a non-smoker and her past medical history included dietcontrolled diabetes and asthma, for which she was taking beclomethasone inhaler twice a day and salbutamol inhaler as required. She had no history of ischemic heart disease or previous history of thrombosis.
Copyright $\odot 2020$ by Thieme Medical Publishers, Inc., 333 Seventh Avenue, New York, NY 10001, USA.

Tel: +1(212) 760-0888.
DOI https://doi.org/ 10.1055/s-0040-1715457. ISSN 0094-6176. 


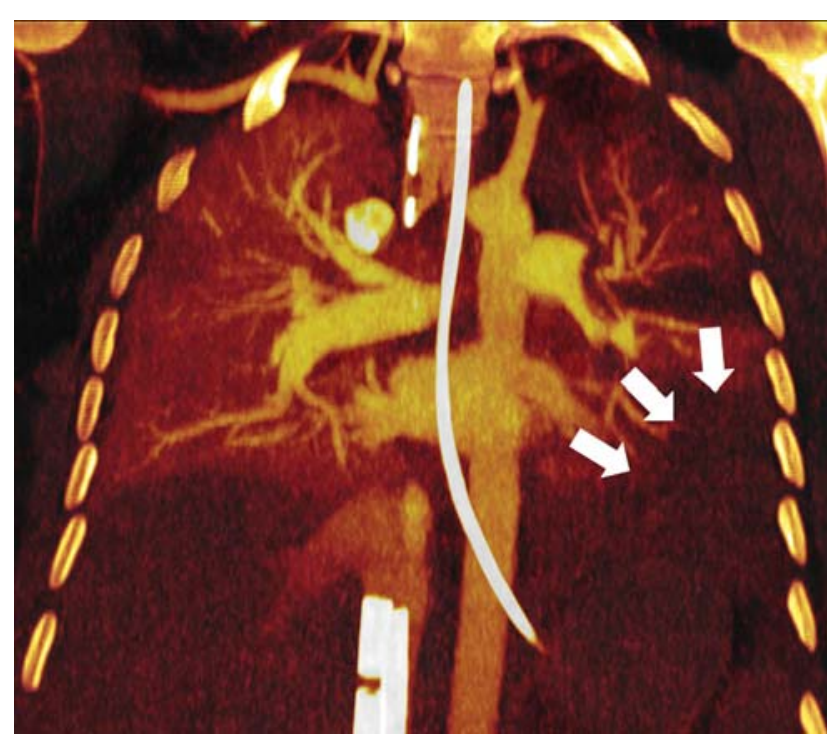

Fig. 1 Maximal intensity projection “iodine map” reflecting blood distribution derived from a dual-energy CT study showing absence of perfusion to the left lung, in particular the lower lobe (arrows); note relatively normal distribution of iodine (reflecting unimpaired perfusion) of the right lung despite extensive consolidation. The ECMO cannulae and nasogastric tube are appropriately positioned (apparent due to their high atomic number constituents).

It is our standard practice to perform an unenhanced computed tomography (CT) of the head to exclude intracranial bleed, prior to starting systemic anticoagulation with unfractionated heparin to prevent thrombosis in the patient and the ECMO circuit. In addition, an "iodine map" dual-energy CT pulmonary angiography (DECTPA) is performed to diagnose pulmonary thrombosis, and assess pulmonary iodine distribution. DECTPA of this patient confirmed a wedge-shaped iodine defect in the left upper lobe and the basal segment of the left lower lobe (-Fig. 1) distal to the pulmonary arteries occluded with thrombus. On admission, blood results showed hemoglobin $83 \mathrm{~g} / \mathrm{L}$ (reference range, $115-151 \mathrm{~g} / \mathrm{L}$ ), platelets $253 \times 10^{9} / \mathrm{L}$ $\left(147-397 \times 10^{9} / \mathrm{L}\right)$, activated partial thromboplastin time 38.0 seconds (26.0-36.0 seconds), prothrombin time $13.5 \mathrm{sec}-$ onds (10.2-13.2 seconds), fibrinogen $4.9 \mathrm{~g} / \mathrm{L}$ (1.5-4.5 g/L), D-dimer 17,597 ng/mL (D-dimer units $[<240 \mathrm{ng} / \mathrm{mL}]$ ), and brain natriuretic peptide (BNP) $608 \mathrm{ng} / \mathrm{L}(<20 \mathrm{ng} / \mathrm{mL}$ ). In view of hemodynamic instability and right ventricular (RV) dysfunction, pulmonary angiography was followed by catheter-directed thrombolysis with alteplase ( $10 \mathrm{mg}$ bolus, then $0.01 \mathrm{mg} / \mathrm{kg}$ / hour for 10 hours) as systemic thrombolysis carries a significant risk of bleeding in patients on ECMO. Following completion of catheter-directed thrombosis with alteplase, unfractionated heparin (12 unit/kg/hour) was started, without a bolus dose, and the dose of heparin gradually titrated to maintain heparin anti-Xa level of 0.3 to $0.5 \mathrm{U} / \mathrm{mL}$ as the patient was on ECMO. On day 1 postthrombolysis, D-dimer was $10,667 \mathrm{ng} / \mathrm{mL}$, and BNP was $137 \mathrm{ng} / \mathrm{L}$, with improved RV function. DECTPA 10 days later showed normalized iodine distribution in the left lung (-Fig. 2) despite only a slight reduction in the lobar thrombus size on CT, suggesting that distal microvascular obstruction had improved. Twenty days after thrombolysis, RV function and estimated

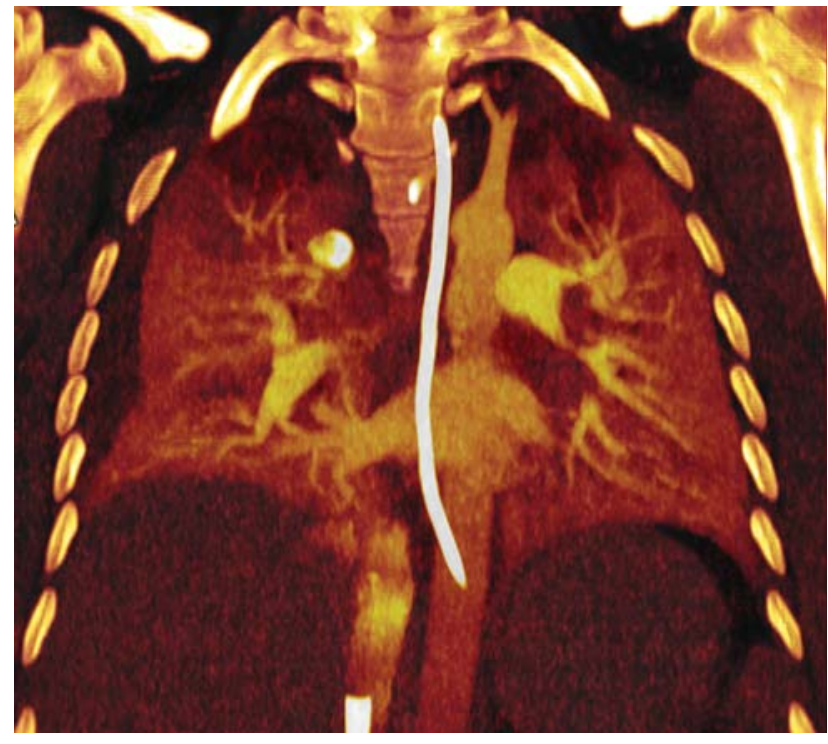

Fig. 2 Surveillance iodine map image 10 days later confirms improved and equal distribution of iodine in both lungs; iodine distribution defects are persistent in both lung apices.

pulmonary artery pressures improved with no hemorrhagic complications. Following a total length of 73 days in the intensive care unit (including 60 days on ECMO), the patient was discharged to their local hospital.

Catheter-directed thrombolysis allows delivery of the thrombolytic agent directly to the area of highest embolic burden via a catheter. The potential advantage of local delivery is a lower required dose of alteplase; therefore, risk of bleeding is lower compared with systemic thrombolysis. In an analysis of a nationwide inpatient care database including 1,521 patients treated with thrombolysis for PE prior to COVID-19, 1,169 patients received systemic thrombolysis (76.9\%) and 352 patients received catheter-directed therapy $(23.1 \%)$. With the propensity-matched comparison, intracranial hemorrhage was significantly lower in the catheter-directed therapy group compared with systemic thrombolysis group ( 21.0 vs. $10.5 \%, p<0.001){ }^{3}$ Patients on ECMO are at additional risk of bleeding, especially intracerebral hemorrhage, due to the effects of ECMO circuit in hemostasis of the patient. These include blood at high shear through the ECMO circuit results in acquired von Willebrand syndrome, which was notably evident in this patient by von Willebrand factor antigen level of $653.5 \mathrm{IU} / \mathrm{dL}$ and von Willebrand factor ristocetin cofactor activity of $133.6 \mathrm{IU} / \mathrm{dL}$ (i.e., activity:antigen ratio of 0.20 ) at the time of thrombolysis. In addition, patients on ECMO develop thrombocytopenia and platelet dysfunction even when the platelet count remain normal, due to loss of platelet surface receptors, and thereby contributing to increased risk of bleeding. ${ }^{4}$ Catheter-directed thrombolysis has been given to patients without COVID-19 receiving $\mathrm{ECMO}^{5,6}$ and patients with COVID-19 not receiving $\mathrm{ECMO}^{7}$ with successful outcomes. However, this is the first reported case in the literature of a patient with COVID-19 pneumonia receiving ECMO treated with catheter-directed 
thrombolysis. Due to the extreme risk of bleeding in patients on ECMO, systemic thrombolysis is not generally recommended, and in such cases catheter-directed thrombolysis as in our case may be lifesaving. The use of DECTPA as surveillance in this patient was highly valuable as the iodine distribution maps depicted an improvement in perfusion that would not have been appreciated using conventional CT pulmonary angiography alone.

\section{Authors' Contributions}

Clinical data collection: all authors; study concepts and design: D.J.A., L.C.P., C.A.R.; literature review: all authors; manuscript preparation/editing: all authors; final manuscript review: all authors.

\section{Funding}

None.

\section{Conflict of Interest}

Authors have no conflict of interests.

\section{Acknowledgments}

The authors are grateful to the Royal Brompton and Harefield Hospitals Charity for financial support in the purchase of dual-energy advanced processing software.

\section{References}

1 Liu Z, Xiao X, Wei X, et al. Composition and divergence of coronavirus spike proteins and host ACE2 receptors predict potential intermediate hosts of SARS-CoV-2. J Med Virol 2020; 92(06):595-601

2 Helms J, Tacquard C, Severac F, et al; CRICS TRIGGERSEP Group (Clinical Research in Intensive Care and Sepsis Trial Group for Global Evaluation and Research in Sepsis). High risk of thrombosis in patients with severe SARS-CoV-2 infection: a multicenter prospective cohort study. Intensive Care Med 2020;46(06): 1089-1098

3 Kuo WT, Gould MK, Louie JD, Rosenberg JK, Sze DY, Hofmann LV. Catheter-directed therapy for the treatment of massive pulmonary embolism: systematic review and meta-analysis of modern techniques. J Vasc Interv Radiol 2009;20(11):1431-1440

4 Arachchillage DRJ, Passariello M, Laffan M, et al. Intracranial hemorrhage and early mortality in patients receiving extracorporeal membrane oxygenation for severe respiratory failure. Semin Thromb Hemost 2018;44(03):276-286

5 Dickens B, Bryant C, Gaillard J, Westphal N. ARDS and massive pulmonary embolism: the combined use of extracorporeal membrane oxygenation (ECMO) with thrombolytics. Case Rep Crit Care 2020;2020:1032629

6 Lauren Lindsey J, Jain R, Vachharajani V. Catheter directed thrombolysis combined with ECMO for massive pulmonary emboli. Respir Med Case Rep 2018;25:6-8

7 Qanadli SD, Gudmundsson L, Rotzinger DC. Catheter-directed thrombolysis in COVID-19 pneumonia with acute PE: thinking beyond the guidelines. Thromb Res 2020;192:9-11 